\title{
Publisher's Note: High-order-harmonic generation from periodic potentials driven by few-cycle laser pulses [Phys. Rev. A 93, 033852 (2016)]
}

Zhong Guan, Xiao-Xin Zhou, and Xue-Bin Bian

(Received 13 April 2016; published 22 April 2016)

DOI: 10.1103/PhysRevA.93.049903

This paper was published online on 28 March 2016 with an error in Fig. 6. Figure 6 has been replaced as of 14 April 2016. The figure is incorrect in the printed version of the journal, therefore for the benefit of the print readership, the figure is replicated below.

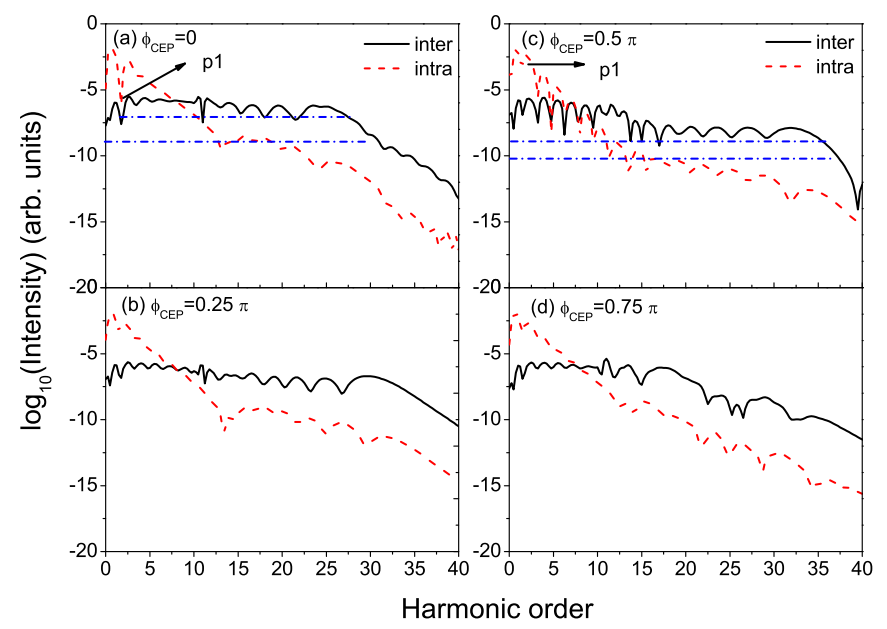

FIG. 6. Interband and intraband current contributions with different CEPs: (a) $\phi_{\mathrm{CEP}}=0$, (b) $\phi_{\mathrm{CEP}}=0.25 \pi$, (c) $\phi_{\mathrm{CEP}}=0.5 \pi$, and (d) $\phi_{\mathrm{CEP}}=0.75 \pi$. Other laser parameters are the same as those in Fig. 5 . 